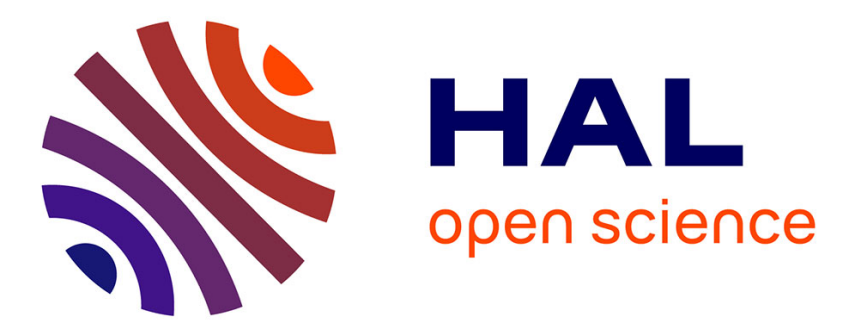

\title{
Fragmentation sociale et Performance du gouvernement de la Cité
}

\author{
Emmanuel Okamba
}

\section{To cite this version:}

Emmanuel Okamba. Fragmentation sociale et Performance du gouvernement de la Cité. Question(s) de Management, 2013, 2, pp.80-94. 10.3917/qdm.131.0079 . hal-01397867

\section{HAL Id: hal-01397867 \\ https://hal.science/hal-01397867}

Submitted on 16 Nov 2016

HAL is a multi-disciplinary open access archive for the deposit and dissemination of scientific research documents, whether they are published or not. The documents may come from teaching and research institutions in France or abroad, or from public or private research centers.
L'archive ouverte pluridisciplinaire HAL, est destinée au dépôt et à la diffusion de documents scientifiques de niveau recherche, publiés ou non, émanant des établissements d'enseignement et de recherche français ou étrangers, des laboratoires publics ou privés. 


\title{
Fragmentation sociale et Performance du gouvernement de la Cité
}

\author{
Par : Emmanuel OKAMBA \\ Maître de Conférences HDR en Sciences de Gestion \\ UFR Sciences Economiques et Gestion \\ Laboratoire Institut de Recherche en Gestion \\ Université de Paris Est, Marne La Vallée \\ 5 Bld Descartes \\ Champs Sur Marne \\ 77454 Marne La Vallée Cedex 02 \\ okamba@univ-mlv.fr
}

\begin{abstract}
Résumé
Cet article analyse le lien entre les stratégies de gestion de la diversité sociale et la performance de la structure des organisations. Il montre que le leadership et la structure du gouvernement de la Cité sont souverains lorsqu'ils sont adaptés aux spécificités structurelles et culturelles de la société dans laquelle, ils prennent racine. Ce déterminisme du lien entre la structure, le leadership et la performance, confirme le postulat de la primauté de la structure par rapport aux phénomènes.
\end{abstract}

\section{Mots clés}

Fragmentation tribale, gouvernance, diversité sociale, structuralisme

\begin{abstract}
This article analyzes the relationship between the management strategies of social diversity and the performance of the structure of organizations. It shows that the leadership and structure of the city government are sovereign when they are tailored to the specific structural and cultural society in which they are rooted. The determinism of the link between structure, leadership and performance, confirms the assumption of the primacy of the structure with respect to the phenomena.
\end{abstract}

\section{Keywords}

Tribal fragmentation, governance, social diversity, structuralism 


\section{Introduction}

Dans son étude sur le leadership du dirigeant des Etats traditionnels du bassin du Congo, G. BALANDIER (1965) constatait qu'il s'agissait d'un "Dirigeant d'exception à double face: brutal et dominateur, en même temps justicier et conciliateur». Ce dirigeant est celui qui prend des décisions raisonnables (Sagesse, en équité (Juste) et en assume les conséquences (Force). Ce leadership mobilisateur ou «souverain», s'accompagne d'une stratégie géocentrique de gestion de la diversité sociale, fondée sur l'intégration à part égale des membres des principaux groupes représentatifs de la diversité sociale dans la composition du gouvernement de la Cité. De nos jours, ce dirigeant d'exception est en voie de disparition, alors que la structure équiproportionnelle traditionnelle de la gouvernance de la Cité, animée par un leadership mobilisateur, continue d'influencer les représentations culturelles de la population dans leurs jeux de fragmentation sociale. Quelle est la nature du lien qui existe entre la structure, le leadership et la performance d'une organisation dans un contexte de diversité sociale et culturelle?

Nous soutenons l'idée de l'existence d'une relation déterministe entre la structure, le leadership et la performance des organisations dans le bassin du Congo. Ce déterministe avait déjà observé dans la société indo-européenne, par G. DUMEZIL (1958) qui constatait la présence d'une structure de gouvernance générique ou souveraine aussi bien dans la mythologie, dans les récits fondateurs de la Rome antique, que dans les pratiques des organisations modernes. La société de l'Ancien Régime se divise en trois classes: Oratores, classe de ceux qui prient (le clergé), Bellatores, classe de ceux qui font la guerre et la noblesse (le roi, les princes, les militaires...), et Laboratores, classe de ceux qui travaillent ou Tiers État (bourgeois, paysans, citoyens...). Dans les institutions d'essence royale, féodale ou démocratique, cette structure mythique opère selon le même processus de «continuité » de la fragmentation sociale, pour reproduire par contrat à tous les échelons de la hiérarchie, le modèle de l'ordonnancement du travail entre les classes sociales. Ce déterminisme structurel a été confirmé par le courant de la contingence culturelle qui étudie l'influence des contraintes culturelles d'une société sur ses organisations. Il montre à travers les travaux de P.D'IRIBARNE (1989) qu'en France, le modèle de la division du travail de l'Ancien Régime entre les emplois nobles détenus par les élites et les emplois les moins nobles détenus par la classe ouvrière, continue à se reproduire dans les organisations marchandes et non marchandes modernes. Même si l'auteur ne montre pas comment chaque groupe est représenté dans la composition du gouvernement de la Cité, la perpétuation de ce modèle mythique de la fragmentation sociale, fondé sur la coopération des classes opposées, résulte de la prégnance des valeurs culturelles de la «méritocratie » ou la «logique de l'honneur » qui régule le système de gouvernance de la Cité et des organisations. Les structures de gouvernement les plus performantes seraient celles qui se rapprochent de ce modèle générique de la société ; alors que les moins performantes seraient celles qui s'en éloignent. M.AOKI (2006) est arrivée à la conclusion dans une approche comparée de la structure des entreprises japonaises avec celle des entreprises américaines et leur performance. Ces travaux parmi tant d'autres, ont confirmé la pertinence du postulat structuraliste de la primauté de la structure sur les phénomènes (C.LEWIS-STRAUSS (1958)), d'après lequel, dans toute société, il existe une structure fondamentale ou souveraine qui organise et anime la conduite de ses organisations indépendamment des phénomènes. La performance d'une structure de gouvernement, dépend donc de son degré d'adaptation au modèle générique ou souverain de la société dont elle est issue.

A partir de l'analyse factorielle des données de 41 gouvernements du Congo, formés par 20 Premiers Ministres (annexe $\mathrm{n}^{\circ} 1$ ), qui ont dirigés 14 plans de développement économique sous le règne de 6 Présidents de la République, du 15 août 1960 jusqu'à nos jours, nous voulons démontrer que la performance d'une structure du gouvernement, dépend de son degré d'adaptation à la structure souveraine du gouvernement de la Cité et de son leadership. Nous présenterons d'abord les enjeux de la fragmentation sociale de la population, à partir de la distribution statistique des postes ministériels entre les principales tribus du Congo (I), avant d'en déduire par un scoring, le degré du lien entre le leadership, la configuration structurelle du gouvernement de la Cité et la performance dans le temps (II).

\section{I- Les enjeux de la fragmentation sociale et du leadership souverain}

La structure du gouvernement des organisations est le produit de la stratégie de fragmentation sociale et culturelle de la population. La fragmentation sociale et culturelle est le processus par lequel une population naturellement composée de groupes sociaux hétérogènes, divise le travail entre les sousensembles plus ou moins homogènes, dans lesquels sont réparties les fonctions de pouvoir, de gestion 
et de contrôle, selon les savoirs, les compétences distinctives et les savoir-être des individus qui les composent. C'est un moyen de lutter contre la ségrégation de jure ou de Droit (instaurée par la loi) et de facto (non instaurée par la loi). La fragmentation sociale de la population est reconnue par les Nations Unies par la résolution 47/135 du 18 décembre 1992 qui exige des Etats membres, la prise en compte des intérêts des minorités raciales, religieuses, linguistiques,... dans le gouvernement de la Cité et des organisations. Les jeux de coopération entre les membres de la diversité sociale, structurent la distribution des postes ministériels, suivant plus ou moins l'ordre de la fragmentation sociale naturelle de la population. Le cas de la distribution des postes ministériels entre les principales tribus du Congo dans les 41 gouvernements, formés depuis 1960, dans le cadre de l'unité nationale fondée sur la disparaître progressivement des inégalités naturelles entre les tribus dans la gouvernance de la Cité, nous sert d'exemple.

I-1-Les modèles de fragmentation sociale: Les économistes du développement ont établi une relation linéaire entre la division ethnique ou tribale de la population d'un pays et son niveau du développement politique (démocratie ou dictature) et économique (industrialisé ou sous industrialisé), à partir de trois modèles qui ont a des affinités avec le déterminisme structurel: essentialiste, évolutionniste et matérialiste.

Le premier modèle part de la structure issue des divisions ethniques pour expliquer la dynamique des évolutions économiques et politiques des pays. Il a été proposé par W.EASTERLY et R.LEVINE (1997) qui ont cherché à mettre en lumière, les effets de la fragmentation ethnique sur les performances économiques par l'économétrie. Leur modèle prend la croissance économique par tête d'habitant comme variable expliquée, et introduit un indice de fragmentation ethnique parmi les variables explicatives liées aux politiques publiques. Ils trouvèrent ainsi une corrélation négative entre le degré de division ethnique et la croissance économique. Le coefficient mesurant l'impact de ces divisions diminue, toutefois, au fur et à mesure qu'on ajoute des variables de politiques publiques dans les variables explicatives. Dans les pays africains, la diversité ethnique manifeste un impact négatif par le biais des politiques publiques, lesquelles, en présence d'une forte hétérogénéité de la population, sont peu enclines à servir l'intérêt général du pays. Alors qu'en Asie, la diversité ethnique manifeste un impact positif. La fragmentation ethnique est alors une cause potentielle de l'explication du retard du développement économique et de l'absence de démocratie en Afrique. Les auteurs en conclurent que l'effet de la diversité ethnique sur la croissance économique des pays africains, passe avant tout par le choix des gouvernants en matière économique (W.EASTERLY, J.RITZEN, et WOOLCOCK M. (2006)).

Le second modèle est développé par K.DEUTSCH (1963) et S-P. HUNTINGTON (1968). Il est fondé sur la « théorie de la modernisation » qui repose sur l'idée selon laquelle, il existe un lien étroit entre le développement socio-économique et certains changements culturels qui touchent au système de valeurs et à l'identité des individus. Le développement économique entrâne une hausse de la productivité du travail, pousse à la spécialisation professionnelle, accroit le niveau d'éducation de la population, et procure un surcroît de bien-être matériel. Il en résulte un accroissement du volume d'échanges entre les individus, une plus grande ouverture aux groupes sociaux distincts, et l'acquisition d'un ensemble de références communes entre les populations géographiquement éloignées, à travers un contrat. Cette évolution des valeurs individuelles entraînera, la disparition progressive des identités traditionnelles et développera une conscience nationale.

Quant au troisième modèle, il considère depuis les travaux de R.BATES (1974) que le développement économique, crée une nouvelle stratification sociale selon les critères qui lui sont propres, et suscite une compétition entre les individus et entre les groupes ethniques pour l'acquisition des «biens désirés» : la richesse, le statut professionnel, le niveau d'éducation et d'urbanité.... L'identité ethnique est alors utilisée par les dirigeants politiques comme le fondement de la constitution des groupes aux intérêts divergents dans la compétition pour la modernité. La fragmentation ethnique est mobilisée dans les projets de développement économique et social comme le principe unificateur efficace des groupes tribaux antagonistes. L'enracinement au pouvoir des leaders des groupes tribaux, résulte alors de leur capacité à extraire des biens et des services du secteur moderne et à satisfaire la demande de leurs membres pour les composantes du modèle du gouvernance de la Cité qui se modernisent. Ce faisant, ils se rendent dispensables. Mais comme le confirme T.BOSSUROY (2006) dans ses travaux sur les déterminants de la fragmentation ethnique en Afrique de l'Ouest : "l'appartenance ethnique détermine l'identité des individus, leurs préférences et leurs choix; comme le groupe social défini par l'ethnie est différent, et généralement plus étroit, que l'ensemble politique auquel les individus appartiennent (leur État ou leur nation), l'attention qu'ils portent aux intérêts particuliers de leur 
ethnie les détourne de l'intérêt général, et affaiblit la construction d'une conscience collective nécessaire au fonctionnement des États ». L'organisation et la régulation de la structure du gouvernement d'une Cité sont alors, le reflet du processus de fragmentation sociale et culturelle dans lequel, les luttes entre les membres de la diversité sociale, améliorent plus ou moins l'utilité économique de leurs membres qui, en se spécialisant dans l'occupation des fonctions ministérielles, stabilisent le système de gouvernance de la Cité dans le temps et l'espace. Dans ce sens, selon JB CARRIERE, M.LEQUIN (2009): "L'émergence du concept de gouvernance cherche à combler l'absence de solutions de la théorie en gestion des organisations, laquelle offre peu de repères pour la coordination d'action inter organisationnelle conçue dans une perspective de réseau. La théorie organisationnelle est centrée sur le développement de modèles d'action et de stratégie dont la référence centrale est l'organisation, alors qu'en gouvernance la référence centrale est la coordination entre organisations. Ce décentrement peut être déstabilisant pour le gestionnaire conventionnel et c'est sur ce point que les repères théoriques lui sont actuellement de peu de soutien pratique». La gouvernance publique peut alors être " comprise comme étant l'établissement de processus, de règles et de structures partagés de gestion, autant informels que formels, facilitant la coordination, la coopération et la complémentarité, à caractère continu et institutionnalisé, entre les gouvernements et les organisations ». Il s'agit essentiellement d'une gouvernance partenariale (T. GARVEY et P.L. SWAN (1994)), fondée sur la coopération des parties prenantes, entre celles qui dirigent (la direction) et celles qui contrôlent la gestion (les autres membres du conseil d'administration) et non sur leur opposition. Cette gouvernance s'oppose à la gouvernance actionnariale ou capitaliste (G.CHARREAUX (1997), G.CHARREAUX et J.P.PITOL-BELIN (1997)), fondée sur l'opposition entre les parties. Dans ce cadre, la dynamique de la gouvernance est fondée sur la résolution de la tension résultant du conflit d'intérêt et de la nécessaire coopération entre les partenaires dans la structure du gouvernement de la Cité comme le montre le cas de la fragmentation tribale de facto du Congo.

I-2-Le cas de la fragmentation matérialiste du Congo: Le Congo compte 4,1 millions d'habitants, répartis sur une superficie de $342000 \mathrm{~km}^{2}$, en 74 ethnies, se rattachant à 3 principaux groupes tribaux dont : au sud du pays, le groupe Bakongo qui représente 51,5\% de la population totale; au centre, le groupe Téké $(17,3 \%)$ et au nord, le groupe Mbosi (11,5\%). A côté de cette triade tribale naturelle, se trouvent des petits groupes tribaux disparates, représentant 19,5\% de la population (Bondjo, Oubanguiens, Sangha, Echiras,...) dans lesquels, émerge un sous groupe que nous dénommons «Oubangui-sangha». L'absence des statistiques ethniques du Congo depuis leur interdiction à partir du recensement de la population de 1974, ne permet pas de savoir avec précision, l'effectif réel de ce groupe émergent. Malgré cette interdiction des statistiques ethniques, la fréquence de la triade tribale (Bakongo, Téké, Mbosi) dans la composition du gouvernement est un indicateur de l'état programmé de l'avancement de la nation vers une société sans discrimination tribale comme le prévoient les dispositions de 1'article 53 de la constitution de 2002, interdisant la préférence tribale ou le tribalisme comme critère de recrutement des membres des partis politiques et de la gouvernance de la Cité. Le tribalisme étant refoulé dans la conscience collective, à travers la promotion de «l'unité » par la classe politique, la distribution des postes ministériels de 41 gouvernements entre les principales tribus, permet de faire le bilan de ce projet sociétal sans discrimination tribale depuis 1960.

I-2-1- La distribution des postes ministériels: Le Tableau n ${ }^{\circ} 1$ montre que de 1960 à 2012, 734 Ministres ont été nommés au Congo dans 41 gouvernements. Soit une moyenne de 18 ministres environ par gouvernement. La structure la moins budgétivore qui a eu l'effectif le plus petit est celle du gouvernement dit des «Technocrates » dirigée du 24/12/1963 au 15/4/1966 par P.LISSOUBA avec 7 Ministres seulement. La structure la plus budgétivore est celle du gouvernement formée par B.KOLELA en 1997 avec 43 ministres. Dans l'effectif de 734 Ministres, 50,43\% sont issus de la tribu Bakongo, contre $20,84 \%$ de Mbosi, $16,76 \%$ de Téké et $12,48 \%$ de la tribu émergente d'Oubanguisangha. Soit:

$$
\Omega=50 \% \text { Bakongo }+16,76 \% \text { Téké }+20,84 \% \text { Mbosi }+12,48 \% \text { Oubangui-Sangha. }
$$

Dans cette structure qui ne suit pas l'ordre de la fragmentation tribale naturelle de la population congolaise, les Mbosi ont occupé majoritairement le poste de Ministre de la Défense et de la Sécurité (37,77\%), les membres de la tribu Oubangui-sangha ont occupé majoritairement à 33,33\%, le poste de Ministre d'Agriculture, des Eaux et Forêts. Les Tékés n'ont occupé majoritairement aucun poste. Tous les autres postes ont été occupés majoritairement par les membres issus de la tribu Bakongo. Cette tendance qui s'observe aussi bien dans le gouvernement d'union nationale que dans le gouvernement ordinaire, montre que le Congo est administré plus par la tribu Bakongo que par les tribus Mbosi et Téké. 
Tableau n ${ }^{\circ}$ : Distribution des postes ministériels selon les tribus de 1960 à 2012

\begin{tabular}{|l|c|c|c|c|c|}
\hline & Bakongo & Mbosi & Téké & Oub-Sangha & Total \\
\hline 1-Primature & $\mathbf{2 5 , 0 0}$ & 10,00 & 4,00 & 1,00 & 40,00 \\
\hline 2-Défense et Sécurité & 12,00 & $\mathbf{1 7 , 0 0}$ & 4,00 & 12,00 & 45,00 \\
\hline 3-Intérieur/Administration du territoire & $\mathbf{3 1 , 0 0}$ & 15,00 & 4,00 & 4,00 & 54,00 \\
\hline 4-Affaires Etrangères/Coopération & $\mathbf{1 5 , 0 0}$ & 14,00 & 9,00 & 3,00 & 41,00 \\
\hline 5-Justice/ travail & $\mathbf{3 2 , 0 0}$ & 18,00 & 6,00 & 5,00 & 61,00 \\
\hline 6-Economie/Finance/ Budget/Plan & $\mathbf{2 4 , 0 0}$ & 11,00 & 7,00 & 16,00 & 58,00 \\
\hline 7-Education/Recherche & $\mathbf{3 1 , 0 0}$ & 11,00 & 25,00 & 6,00 & 73,00 \\
\hline 8-Santé/Affaires sociales & $\mathbf{4 6 , 0 0}$ & 8,00 & 7,00 & 2,00 & 63,00 \\
\hline 9-Agriculture, Eaux et Forêts & 21,00 & 7,00 & 10,00 & 19,00 & 57,00 \\
\hline 10-Industrie/Commerce/Mines/Hydrocarbures & $\mathbf{6 2 , 0 0}$ & 19,00 & 5,00 & 8,00 & 94,00 \\
\hline 11-Transports/Construction/Habitat & $\mathbf{2 2 , 0 0}$ & 5,00 & $\mathbf{2 1 , 0 0}$ & 2,00 & 50,00 \\
\hline 12-Communication/Information/PTT & $\mathbf{2 5 , 0 0}$ & 4,00 & 12,00 & 7,00 & 48,00 \\
\hline 13-Culture/Arts/Sports & $\mathbf{2 1 , 0 0}$ & 14,00 & 9,00 & 6,00 & 50,00 \\
\hline Total & 367,00 & 153,00 & 123,00 & 91,00 & 734,00 \\
\hline \% & $50,00 \%$ & $20,84 \%$ & $16,76 \%$ & $12,40 \%$ & $100,00 \%$ \\
\hline
\end{tabular}

Le «gouvernement ordinaire » est celui issu d'un accord électoral entre les leaders des différentes tribus pour constituer une majorité présidentielle autour d'un leader politique qui a conquis le pouvoir. Elle représente $48,78 \%$ de l'effectif total des gouvernements du Congo (Tableau n ${ }^{\circ}$ ). Sur la moyenne des 13 postes les plus promus dans ce type de gouvernement, regroupant 414 ministres, la structure du gouvernement de la Cité $(\Omega)$ a la forme suivante:

$\Omega=52,64 \%$ Bakongo $+18,06 \%$ Téké $+18,50 \%$ Mbosi $+10,79 \%$ Oubangui-Sangha.

Dans cette structure, les Bakongo ont occupé majoritairement presque tous les postes ministériels, sauf la Défense et la Sécurité qui ont été occupés majoritairement $(45,83 \%)$ par les membres de la tribu Oubangui-Sangha, et de la Culture, des Arts et des Sports qui ont été occupés majoritairement par les Mbosi (34,48\%). Le Transport et l'Habitat ont été occupés majoritairement par les Téké $(55,17 \%)$. Dans le gouvernement ordinaire, la distribution des postes ministériels entre les membres de la diversité sociale suit presque l'ordre de la fragmentation tribale naturelle. C'est une structure ethnocentrique.

Le « gouvernement d'union nationale » ou de sortie de crise est celui formé pour résoudre une crise politique majeure (assassinat ou démission du Président de la République, censure du gouvernement par l'opposition, grève générale, guerre civile....). Elle regroupe l'ensemble des leaders des forces politiques de l'opposition et de la majorité y compris ceux de la société civile. Elle représente 57,50\% des structures gouvernementales formées au Congo (Tableau n ${ }^{\circ} 3$ ). Sur les 13 postes les plus promus dans cette configuration, regroupant un effectif total de 280 ministres, la structure du gouvernement se présente de la manière suivante:

$\Omega=45,71 \%$ Bakongo $+25,36 \%$ Mbosi $+13,93 \%$ Téké et $15,00 \%$ Oubangui-sangha.

Cette structure ne suit pas l'ordre de la fragmentation tribale naturelle de la population. Comme dans la structure précédente, les Ministres d'origine Bakongo ont occupé majoritairement tous les postes ministériels, sauf les Affaires Etrangères et la Coopération majoritairement occupés par les Téké $(35 \%)^{1}$ et l'Agriculture, les Eaux et Forêts qui ont été occupés majoritairement par les Ministres issus de l'Oubangui-sangha (45\%). Le gouvernement d'union nationale a une structure de type géocentrique, c'est à dire formée en tenant compte de l'équilibre des forces politiques en jeu sur le territoire.

Ainsi, durant la période étudiée, les stratégies de fragmentation sociale des leaders politiques se sont traduites par le passage du gouvernement ordinaire vers le gouvernement d'union nationale. $\mathrm{Ce}$ passage se solde par un gain de 173 postes ministériels, grâce à la perte des postes ministériels par la tribu Bakongo de l'ordre de 6,93\%, contre 4,57\% pour la tribu Téké. La tribu Mbosi a gagné 7,30\% de postes ministériels et la tribu Oubangui-Sangha a gagné $4,21 \%$ de postes (Tableau n ${ }^{\circ} 4$ ).

\footnotetext{
${ }^{1}$ Les Téké sont les descendants du roi Makoko qui signa le principal protectorat avec la France le 10 septembre 1880 représentée par Pierre Savorgnon de Brazza, et qui fit du Congo une colonie française jusqu'au 15 août 1960.
} 
Tableau $\mathrm{n}^{\circ} 2$ : La distribution des 13 postes ministériels les plus promus dans le gouvernement ordinaire entre les tribus de 1960 à 2012

\begin{tabular}{|c|c|c|c|c|c|}
\hline & Bakongo & Mbosi & Téké & Oub-Sangha & Total \\
\hline 1-Primature & 13 & 5 & 1 & & 19 \\
\hline 2-Défense et Sécurité & 8 & 2 & 3 & 11 & 24 \\
\hline 3-Intérieur /Administration du territoire & 20 & 9 & 4 & & 33 \\
\hline 4-Affaires Etrangères/Coopération & 11 & 8 & 1 & & 20 \\
\hline 5-Justice/ travail & 19 & 12 & 5 & 4 & 40 \\
\hline 6-Economie/Finance/ Budget/Plan & 15 & 6 & 4 & 12 & 37 \\
\hline 7-Education/Recherche & 25 & 3 & 19 & 5 & 52 \\
\hline 8-Santé/Affaires sociales & 35 & 3 & 3 & 1 & 42 \\
\hline 9-Agriculture, Eaux et Forêts & 14 & 6 & 7 & 9 & 36 \\
\hline 10-Industrie/Commerce/Mines/Hydrocarbures & 48 & 12 & 5 & 3 & 68 \\
\hline 11-Transports/Construction/Habitat & 9 & 3 & 16 & 1 & 29 \\
\hline 12-Communication/Information/PTT & 14 & 3 & 8 & & 25 \\
\hline 13-Culture/Arts/Sports & 8 & 10 & 8 & 3 & 29 \\
\hline Total & 239 & 82 & 84 & 49 & 454 \\
\hline$\%$ & $52,64 \%$ & $18,06 \%$ & $18,50 \%$ & $10,79 \%$ & $100,00 \%$ \\
\hline
\end{tabular}

Les tribus naturellement minoritaires ont gagné davantage de postes ministériels durant la période étudiée. Les membres des tribus naturellement majoritaires (Bakongo et Téké) ont donc intérêt à voir se former au Congo plus de gouvernements ordinaires que d'union nationale, alors que ceux des tribus naturellement minoritaires (Mbosi, Oubangui-sangha et autres) ont intérêt à voir formé davantage de gouvernements d'union nationale. La tension qui en résulte dans le choix stratégique du type de gouvernement, se traduit par la naissance d'une oligarchie politique instable, composée selon la loi de Pareto d'environ $20 \%$ de la population qui concentre plus de $80 \%$ de la richesse du pays; alors que les $80 \%$ de la population qui restent, ne bénéficient que de $20 \%$ de la richesse de la nationale. D'où:

- l'instabilité constitutionnelle:14 textes constitutionnels entre 1960 et 2002, soit un texte tous les 3,14 ans en moyenne. Dans ces textes $42,86 \%$ sont des Actes fondamentaux pris pour sortir des crises politiques contre $57,14 \%$ qui sont des Constitutions;

Tableau n ${ }^{\circ}$ : La distribution des 13 postes les plus promus dans les gouvernements d'union nationale entre les tribus de 1960 à 2012

\begin{tabular}{|l|c|c|c|c|c|}
\hline & Bakongo & Mbosi & Téké & Oub-Sangha & Total \\
\hline 1-Primature & $\mathbf{1 2}$ & 5 & 3 & 1 & 21 \\
\hline 2-Défense et Sécurité & 4 & $\mathbf{1 5}$ & 1 & 1 & 21 \\
\hline 3-Intérieur/Administration du territoire & $\mathbf{1 1}$ & 6 & & 4 & 21 \\
\hline 4-Affaires Etrangères/Coopération & 4 & 6 & $\mathbf{8}$ & 3 & 21 \\
\hline 5-Justice/ travail & $\mathbf{1 3}$ & 6 & 1 & 1 & 21 \\
\hline 6-Economie/Finance/ Budget/Plan & $\mathbf{9}$ & 5 & 3 & 4 & 21 \\
\hline 7-Education/Recherche & $\mathbf{6}$ & $\mathbf{8}$ & 6 & 1 & 21 \\
\hline 8-Santé/Affaires sociales & $\mathbf{1 1}$ & 5 & 4 & 1 & 21 \\
\hline 9-Agriculture, Eaux et Forêts & 7 & 1 & 3 & $\mathbf{1 0}$ & 21 \\
\hline 10-Industrie/Commerce/Mines/Hydrocarbures & $\mathbf{1 4}$ & 7 & & 5 & 26 \\
\hline 11-Transports/Construction/Habitat & $\mathbf{1 3}$ & 2 & 5 & 1 & 21 \\
\hline 12-Communication/Information/PTT & $\mathbf{1 1}$ & 1 & 4 & 7 & 23 \\
\hline 13-Culture/Arts/Sports & $\mathbf{1 3}$ & 4 & 1 & 3 & 21 \\
\hline Total & 128 & 71 & 39 & 42 & 280 \\
\hline \% & $45,71 \%$ & $25,36 \%$ & $13,93 \%$ & $15,00 \%$ & $100,00 \%$ \\
\hline
\end{tabular}

- l'instabilité gouvernementale: la durée de vie moyenne d'un gouvernement n'est que de 1,33 an;

- l'instabilité du régime politique : le régime présidentiel représente $82,86 \%$ des gouvernements contre $17,14 \%$ du régime parlementaire, alors que $75 \%$ des Présidents ont acquis le pouvoir par un coup d'Etat tribal contre $25 \%$ qui ont été élus. D'où la difficulté de tendre vers une gestion de la diversité 
sociale performante sans préférence tribale, tant que le niveau de vie de chaque citoyen ne s'améliore pas de manière satisfaisante.

Le régime présidentiel se caractérise par la séparation stricte des pouvoirs législatif et exécutif, avec la nomination d'un Premier Ministre, distinct du Président de la République. Chaque Ministre est responsable de son action devant le Président de la République. Les membres du gouvernement sont responsables collectivement et politiquement devant le pouvoir législatif. L'exécutif et le législatif se contrôlent mutuellement.

Tableau n ${ }^{\circ}$ 4: Ecarts des postes ministériels entre les tribus de 1960 à 2012

\begin{tabular}{|c|c|c|c|c|c|}
\hline & Bakongo & Mbosi & Téké & Oub-Sangha & Total \\
\hline 1-Primature & -1 & $\mathbf{0}$ & 2 & 1 & 2 \\
\hline 2-Défense et Sécurité & -4 & 13 & -2 & -10 & -3 \\
\hline 3-Intérieur /Administration du territoire & -9 & -3 & -4 & 4 & -12 \\
\hline 4-Affaires Etrangères/Coopération & -7 & -2 & 7 & 3 & 1 \\
\hline 5-Justice/ travail & -6 & -6 & -4 & -3 & -19 \\
\hline 6-Economie/Finance/ Budget/Plan & -6 & -1 & -1 & -8 & -16 \\
\hline 7-Education/Recherche & -19 & 5 & -13 & -4 & -31 \\
\hline 8-Santé/Affaires sociales & -24 & 2 & 1 & $\mathbf{0}$ & -21 \\
\hline 9-Agriculture, Eaux et Forêts & -7 & -5 & -4 & 1 & -15 \\
\hline 10-Industrie/Commerce/Mines/Hydrocarbures & -34 & -5 & -5 & 2 & -42 \\
\hline 11-Transports/Construction/Habitat & 4 & -1 & -11 & $\mathbf{0}$ & -8 \\
\hline 12-Communication/Information/PTT & -3 & -2 & -4 & 7 & -2 \\
\hline 13-Culture/Arts/Sports & 5 & -6 & -7 & $\mathbf{0}$ & -8 \\
\hline Total & -111 & -11 & -45 & -7 & -174 \\
\hline$\%$ & $-6,93 \%$ & $7,30 \%$ & $-4,57 \%$ & $4,21 \%$ & $0,00 \%$ \\
\hline
\end{tabular}

Mais le règne du parti unique de 1963 à 1992 au Congo, invite à relativiser ce postulat, car, durant cette période, seul le Comité Central du Parti-Etat (Conseil National de la Révolution (1963-1968) ou le Parti Congolais du Travail (PCT) (1968-1992) du parti unique pouvait dissoudre le Parlement (Assemblée Nationale Populaire), dès lors que les délibérations de ce dernier n'étaient plus conformes aux orientations du Parti-État. Mais le Parlement ne pouvait dissoudre le Comité Central du PCT. La censure du gouvernement par l'Assemblée Nationale, le 17 novembre 1992, a réveillé les réflexes autocratiques et génocidaires de la clan au pouvoir. Le régime parlementaire ne peut générer de performance durable de la gouvernance de la Cité, sans le respect de l'éthique morale par les dirigeants politiques.

Le régime parlementaire, est celui dans lequel, le pouvoir exécutif (le gouvernement et le Président de la République) et le pouvoir législatif (l'Assemblée Nationale et le Sénat), dépendent l'un de l'autre. L'exécutif étant responsable devant le Parlement de l'action du gouvernement, mais il peut aussi procéder à sa dissolution. Au Congo, ce régime a eu deux caractéristiques principales:

- le Président de la République exerce également les fonctions de Premier Ministre, de Ministre de la Défense et/ou de la Sécurité, comme ce fut le cas dans les gouvernements de Fulbert YOULOU (19581963), de Marien NGOUABI (1969-1971), de Denis SASSOU-NGUESSO (1997-2002) et d'André MILONGO (Premier Ministre de 1991-1992) qui exerça en plus la fonction de Ministre des Hydrocarbures durant le régime de Transition, issu de la Conférence Nationale Souveraine de 1991;

- le Parlement ne peut dissoudre l'Exécutif. Ces deux principes, montrent que le régime politique majoritaire du Congo est un régime semi-présidentiel qui, généralement est mis en place pour résoudre temporairement une crise politique, sociale et morale majeure. A long terme, le régime semiprésidentiel ne peut générer une performance durable de la gouvernance de la Cité, sans le contrôle réciproque, effectif et permanent entre le pouvoir Exécutif et le pouvoir Législatif au sommet de l'État. I-2-3- La Primature: Le Premier Ministre est chargé de coordonner l'action du gouvernement d'un pays. De 1960 à nos jours, le Congo a connu au moins 20 Premiers Ministres qui ont formé 41 gouvernements (Tableau $\mathrm{n}^{\circ}$ ). $58,97 \%$ des ces gouvernements sont dépourvus de Ministres d'Etat, contre $41,03 \%$ qui en sont pourvus. Sur les 41 Gouvernements étudiés, $64,10 \%$ ont été dirigés par des Premiers Ministres d'origine Bakongo, 23,08\% par des Mbosi, 10,26\% par des Téké et 5,12\% par des Oubangui-sangha (Tableau n ${ }^{\circ}$ ). $48,71 \%$ ont été dirigés par les Administrateurs civils, $38,46 \%$ par les Militaires, 7,69\% par les enseignants et 5,13\% par un Prêtre (Abbé F.YOULOU). 
La distribution des postes de Premier Ministre par tribu est nuancée selon le type de gouvernement. Dans les gouvernements d'union nationale, la distribution des postes de Premiers Ministres profite davantage aux tribus minoritaires qu'aux tribus majoritaires. 57,14\% des Chefs du gouvernement ont été d'origine Bakongo, 23,81\% d'origine Mbosi, 14,29\% d'origine Téké, et 4,76\% d'origine Oubangui-sangha. Ces proportions par tribu sont au-delà de la moyenne générale dans le gouvernement ordinaire avec respectivement $68,42 \%, 26,32 \%, 5,26 \%$ et $0 \%$. Le gouvernement d'union nationale tend à réduire les inégalités naturelles entre les tribus, alors que le gouvernement ordinaire tend à les accentuer même au niveau de la primature.

Tableau n ${ }^{\circ}$ 5: distribution du poste de Premier Ministre entre les tribus principales

\begin{tabular}{|l|c|c|c|}
\hline & Unité Nationale (I) & Ordinaire (II) & Ecart =I-II \\
\hline Bakongo & $57,14 \%$ & $68,42 \%$ & $-11,28 \%$ \\
\hline Mbosi & $23,81 \%$ & $26,32 \%$ & $-2,51 \%$ \\
\hline Téké & $14,29 \%$ & $5,26 \%$ & $9,02 \%$ \\
\hline Oubangui-Sangha & $4,76 \%$ & $0,00 \%$ & $4,76 \%$ \\
\cline { 2 - 4 } & $100,00 \%$ & $100,00 \%$ & $0,00 \%$ \\
\hline
\end{tabular}

I-2-3- La Présidence de la République: Le Président de la République est chargé de diriger le pays. De 1960 à nos jours, le Congo a été dirigé par 6 Présidents de la République (Tableau nº). Le record des mandats revient à Denis SASSOU-NGUESSO (Mbosi) qui a dirigé le pays trois fois (du 17 mars 1977 au 21 avril 1977); du 5 février 1979 au 15 août 1992 et 15 octobre 1997 à nos jours). Deux Présidents ont été élus au suffrage universel direct (Pascal LISSOUBA (1992-1997), ethnie Nzabi, tribu Téké et Denis SASSOU-NGUESSO III (2002 à nos jours). Un seul Président a été élu au suffrage universel indirect par le Parlement (Alphonse MASSAMBA-DEBAT (1963-1968)). L'Abbé Fulbert YOULOU (1960-1963) avait été nommé comme Président de la République par l'ancienne métropole au moment de l'indépendance du Congo, en sa qualité de Premier Ministre de la République naissante.

Sur les 6 Présidents du Congo: 50\% ont été des militaires (3 Mbosi), 33\% des enseignants (1 Bakongo et 1 Téké) et $17 \%$ un ecclésiastique (Bakongo). Excepté J.YHOMBI-OPANGO (Mbosi), Président de transition, les Présidents issus des Chefferies Mbosi ont une longévité cumulée au pouvoir plus importante (39,50 ans) que les Présidents issus des royaumes des Téké et des Bakongo (13,18 ans). Cela s'explique dans une certaine mesure par le fait que dans la Chefferie des Mbosi, les hommes sont initiés à l'exercice du leadership mobilisateur dès l'âge de 7 ans dans une société secrète dénommée Kébé-kébé, fondée sur le culte du serpent Odi, sorte de cobra royal, auteur du monde manifesté. Cette société est une synthèse des valeurs morales des deux sociétés secrètes des hommes, Ondinga ou Okani (Sagesse ) qui forme à l'art de prendre les décisions raisonnables, en équité (Justice ou Otwèrè) avec courage (Force ou réalisation des actions nobles, le Kébé-kébé lui-même). Les initiations aux autres degrés du Kébé-kébé se font à 15, 30, 45 ans. Elles renforcent l'aptitude de l'homme au leadership mobilisateur tout au long de sa vie, afin qu'il devienne un chef de famille, du village ou territoire comme un Yombi ou chef du Kébé-kébé (E.OKAMBA (2012)). Dans les royaumes (Kongo et Téké), la formation du roi au leadership mobilisateur n'est faite qu'au moment de son intronisation par la société Lemba, fondée sur le culte de la panthère chez les Bakongo ou par la société Nkoué-Mbali, fondée sur le culte du serpent chez les Téké. La formation du leadership souverain tout au long de la vie de l'homme est déterminante dans la longévité au pouvoir du leader politique dans le bassin du Congo.

GOLEMAN, D., BOYATZIS, R. \& MCKEE A. (2002) ont montré l'efficacité du leadership mobilisateur par rapport aux autres types de styles de leadership, notamment dans sa capacité à inciter à prendre des décisions raisonnables, à clarifier la situation par l'équité et à respecter l'éthique par le courage du leader (Tableau $\mathrm{n}^{\circ} 7$ ). Le Kébé-kébé qui repose sur le mythe de l'immortalité ou de la réalisation par le dirigeant d'exception des œuvres qui marquent à jamais la conscience collective, est fondé sur l'émulation des talents. Il enseigne la maîtrise de l'équilibre ou de l'harmonie des forces opposées dans toute forme de construction par la maîtrise des proportions y compris dans la composition de la structure du gouvernement de la Cité. 
Tableau n ${ }^{\circ}$ : Longévité des Présidents de la République du Congo au pouvoir depuis 1960

\begin{tabular}{|l|c|c|c|c|c|c|c|c|}
\cline { 2 - 8 } \multicolumn{1}{c|}{} & $\begin{array}{c}\text { Début } \\
\text { mandat }\end{array}$ & Fin mandat & Durée mandat (ans) & Tribu & Métier & $\begin{array}{c}\text { Mode accès } \\
\text { Pouvoir }\end{array}$ & $\begin{array}{c}\text { Cause de départ } \\
\text { du pouvoir }\end{array}$ & Parti politique \\
\hline F.YOULOU & $15 / 08 / 1960$ & $15 / 08 / 1963$ & 3,00 & Bakongo & Abbé & CE & Démission & UDDIA \\
\hline A.MASSAMBA-DEBAT & $19 / 08 / 1963$ & $04 / 09 / 1968$ & 5,05 & Bakongo & Instituteur & ESUI & Démission & MNR \\
\hline M.NGOUABI & $04 / 09 / 1968$ & $18 / 03 / 1977$ & 8,54 & Mbosi & Militaire & CE & Assassinat & PCT \\
\hline D.SASSOU-NGUESSO I & $18 / 03 / 1977$ & $03 / 04 / 1977$ & 0,04 & Mbosi & Militaire & CE & CE & PCT \\
\hline J;YHOMBI-OPANGO & $03 / 04 / 1977$ & $05 / 02 / 1979$ & 1,84 & Mbosi & Militaire & CE & PCT & Défaite Electorale \\
\hline D.SASSOU-NGUESSO II & $05 / 02 / 1979$ & $31 / 08 / 1992$ & 13,58 & Mbosi & Militaire & CE & PCT \\
\hline P.LISSOUBA & $31 / 08 / 1992$ & $15 / 10 / 1997$ & 5,13 & Téké & Universitaire & ESUD & Défaite Guerrière & UPADS \\
\hline D.SASSOU-NGUESSO III & $15 / 10 / 1997$ & & & Mbosi & Militaire & Victoire Guerrière & & PCT \\
\hline
\end{tabular}

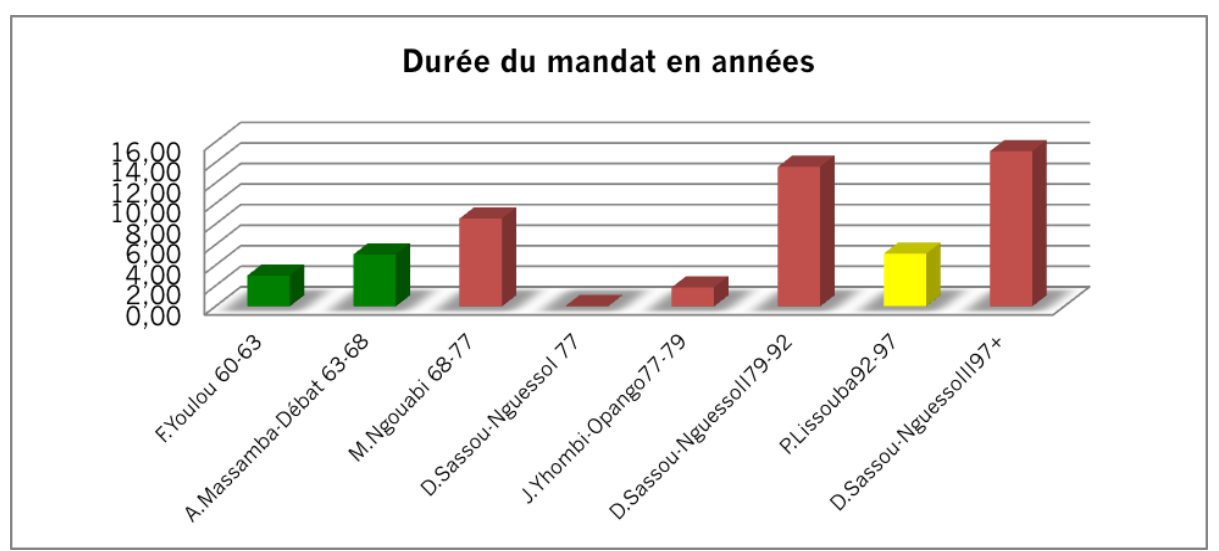

$C E=$ Coup d'Etat $;$ ESUI = Elu au Suffrage Universel Indirect $;$ ESUD = Elu Suffrage au Universel Direct

UDDIA = l'Union pour la Défense Démocratique des Intérêts Africains; PCT = Parti Congolais du Travail, UPADS = l'Union Panafricaine pour la Démocratie Sociale $; M N R=$ Mouvement National de la Révolution

\section{II-Les configurations structurelles du gouvernement}

Distinguons les canons structurant la configuration générique ou traditionnelle du gouvernement de la cité de ceux formant les structures issues des stratégies de fragmentation sociale développées par les partenaires de la gouvernance de la Cité.

\section{II-1- La structure traditionnelle du gouvernement de la Cité du Congo est équiproportionnelle}

Traditionnellement, la société congolaise est fondée sur la promotion de l'idéal de l'équilibre des forces sociales et politiques qui la composent, pour tendre vers une société sans discrimination tribale, dans laquelle le leadership du dirigeant pourvoie une structure équiproportionnelle du gouvernement de la cité et des organisations. Dans cette structure, toutes les tribus importantes majoritaires, sont représentées à part égale plus le souverain lui-même. Cette structure s'observe de manière:

-au sud du pays chez les Bakongo, dans le conseil royal de l'ex-royaume du Kongo (VIIe siècle 1665 ), où quatre clans principaux dirigeaient l'Etat de plus de $2.500 .000 \mathrm{~km}^{2}$ : le clan de Nzinga détenteur du pouvoir royal, celui de Nsaku détenteur du pouvoir judiciaire et prophétique, et celui de Mpanzu détenteur du pouvoir économique, guerrier et sacerdotal et le clan Lukeni, incarnant le pouvoir féminin. Chaque clan était représenté par trois membres au conseil royal plus le roi lui-même. Au total une structure de gouvernement de la Cité de 13 membres (R.BATSIKAMA (1999)). Soit :

$$
\Omega=(3 \text { membres x } 4 \text { principaux clans })+1 \text { membre. }
$$

- au centre, le conseil royal des Téké, formé par 7 membres, choisis parmi les six branches familiales royales (Impio, Isou, Onzala, Impan, Inkiri, Onkonsan), regroupées par trois plus le roi lui-même (E. MOUAYINI OPOU (2005)). Soit:

$$
\Omega=(2 \text { membres } \times 3 \text { branches familiales royales })+1 \text { membre. }
$$

- au nord, en pays Mbosi, le conseil du département mis en place durant la colonisation française (1880-1960), était calquée sur la structure du conseil de la chefferie de 13 membres (J. LOMBARD (1967)), composée de trois chefs choisis dans les trois conseils principaux (le Conseil du village qui regroupe les chefs de familles; le Conseil de terre qui regroupe les chefs de villages ; le Conseil de la 
tribu qui regroupe les principaux chefs de terre). Ces chefs sont assistés par quatre membres. Le conseil du département étant dirigé par le Commandant (français). Soit :

$$
\Omega=(3 \text { chefs x } 4 \text { représentants })+1 \text { représentant. }
$$

Tableau n ${ }^{\circ}$ : Le leadership mobilisateur ou souverain

\begin{tabular}{|l|l|l|l|l|l|l|}
\hline & Autoritaire & Exemple & Coopératif & Participatif & Mentor & Mobilisateur \\
\hline Clarté & - & -- & +++ & +++ & +++ & ++++ \\
\hline Ethique & + & -- & +++ & ++ & +++ & +++ \\
\hline Responsabilité & --- & + & + & ++ & + & ++ \\
\hline Flexibilité & -- & - & ++ & ++ & + & +++ \\
\hline Incitation & - & -- & ++++ & ++++ & ++++ & +++++ \\
\hline Engagement & - & -- & +++ & ++ & ++ & +++ \\
\hline Impact général & Très négatif & Négatif & Positif & Positif & Positif & Très positif \\
\hline Position & $6^{e}$ & $5^{e}$ & $2^{e}$ & $3^{e}$ & $4^{e}$ & $1^{\text {er }}$ \\
\hline
\end{tabular}

D'après GOLEMAN, D., BOYATZIS, R. \& MCKEE A. (2002)

Même si le royaume et la chefferie n'ont pas d'emprise réelle sur la République, dans les représentations culturelles collectives des congolais, ces Etats traditionnels servent toujours de modèle de référence d'organisation parfaite de la Cité, avec un gouvernement comportant un effectif compris entre 7 et 13 membres. Durant la période étudiée, seule la structure du gouvernement de 7 membres mise en place par P. LISSOUBA dite « gouvernement des technocrates », est équiproportionnelle. Elle a intégré complètement et harmonieusement, la triade tribale naturelle dans sa composition. Elle comptait deux ministres par chacune des trois principales tribus plus un ministre issu d'une tribu minoritaire. Soit :

$$
\Omega=(2 \text { ministres } \times 3 \text { tribus })+1 \text { ministre }
$$

Cette structure dans laquelle, le Président de la République assumait la fonction de Ministre de la Défense, est historiquement la plus en phase avec le modèle traditionnel du gouvernement de la Cité du Congo. Son harmonie s'observe sur trois plans : idéologique, technique et de l'efficacité. Sur le plan idéologique, les 7 ministres étaient tous proches de l'éthique protestante et réformiste, même si 43\% parmi eux étaient de tendance libérale et conservatrice, contre $29 \%$ des technocrates sans étiquette politique (société civile) et $28 \%$ qui étaient de tendance progressiste socialisante. Selon M.WEBER (1904), l'éthique protestante a pour but de gagner de l'argent, toujours plus d'argent en se gardant des jouissances strictement de la vie. Cette éthique a favorisé le développement du capitalisme en Allemagne. Elle a trouvé un écho favorable dans la culture de l'Excellence des Bantou. Mais faute de leadership souveraine, au lieu de favoriser un capitalisme privé, le «gouvernement des technocrates» a favorisé un capitalisme d'Etat par la création de plus d'une dizaine d'entreprises publiques, essentiellement avec le soutient financier et technologique de la coopération chinoise. Ces « usines clef en main » installées sans transfert effectif de technologie de la Chine vers le Congo, sont venues grossir le sombre tableau des innombrables «éléphants blancs » de la coopération du Congo avec les pays développés (J.BOKILO (2012)). Or, la Chine qui exige systématiquement dans ses contrats industriels avec les pays développés, une clause de transfert de technologie, pour lui permettre d'être autonome vis à vis de ses partenaires, n'applique pas cette clause dans ses transactions avec les pays en développement. Faute d'exiger cette clause de transfert effectif de technologie, les autorités congolaises établissent depuis cette époque, des contrats industriels avec la Chine et les autres partenaires qui génèrent des "éléphants blancs », dont l'issue est la liquidation à très court terme. Sur le plan technique, ces ministres étaient tous des civils dont $42 \%$ étaient des spécialistes des Services Administratifs et Financiers, 29\% étaient des enseignants et les 29\% autres étaient titulaires d'un doctorat. Avec une durée 2,31 ans, cette structure se situe largement au dessus de la durée moyenne générale de 1,40 an des gouvernements du Congo depuis l'indépendance. Sa performance dans la gestion des plans stratégiques de développement est sans précédente dans l'histoire politique et économique du Congo (Tableau $\mathrm{n}^{\circ} 8$ ). Mais, faute de leadership souverain ou mobilisateur, ce gouvernement a très vite sombré dans la violence politique et a précipité la chute de l'ensemble de 
l'édifice du socialisme bantou ou socialisme à l'africain, celui fondé sur la lutte des tribus que pourvoyait le pouvoir Exécutif.

La structure équiproportionnelle s'observe dans les gouvernements d'autres pays africains, notamment dans les Etats nés de l'éclatement de l'Empire du Ghana, dans la fédération du Mali (7,45 millions d'habitants et 1.436.190 $\mathrm{km}^{2}$ en 1960), constituée par le Soudan français (Mali) et le Sénégal, reconnu par la France, le 4 avril 1959. La première assemblée fédérale de cette fédération était composée de 20 membres élus par pays. Léopold Sédar Senghor en sera élu Président, alors que Modibo Keïta en deviendra, le Chef du gouvernement fédéral formé le 5 avril 1959. Ce gouvernement était constitué à parité égale de maliens et de sénégalais comme l'indique le Tableau $n^{\circ} 9$. L'indépendance de la fédération du Mali fut déclarée le 20 juin 1960, après le transfert des compétences par la France. Mais les dissensions opposèrent les Senghor et Modibo dans l'exercice du leadership au sein de la fédération. Le 20 août 1960, le Sénégal proclama son indépendance et Senghor sera élu Président de la République, le 5 septembre 1960. Cette fédération a été une structure de transition des différentes colonies françaises d'Afrique concernées vers des Etats souverains.

Tableau n ${ }^{\circ}$ : Bilan des plans de développement économique, social et culturel réalisés depuis $1961^{2}$

\begin{tabular}{|c|c|c|c|c|}
\hline Année & Nom du Plan & $\begin{array}{l}\text { Montant en } \\
\text { milliards de } \\
\text { FCFA) }\end{array}$ & $\begin{array}{c}\text { Financement } \\
\text { externe }\end{array}$ & $\begin{array}{c}\text { Réalisation en } \\
\%\end{array}$ \\
\hline $1961-1963$ & Plan triennal de développement & 38 & $55,26 \%$ & ND \\
\hline $1964-1968$ & $\begin{array}{l}1^{\text {er }} \text { Plan quinquennal de développement } \\
\text { économique }\end{array}$ & 62,2 & $72,87 \%$ & $98,11 \%$ \\
\hline $1975-1977$ & Programme triennal & 75,47 & & $37 \%$ \\
\hline $1982-1986$ & $\begin{array}{lrlr}\text { Plan quinquennal } & \text { de } & \text { développement } \\
\text { économique et social } & & \\
\end{array}$ & 1.108 & & $67 \%$ \\
\hline $1986-1990$ & Plan d'ajustement structurel & 511,065 & $100 \%$ & $100 \%$ \\
\hline $1990-1994$ & $\begin{array}{l}\text { Plan d'ajustement économique et social } \\
\text { (PAES) }\end{array}$ & & $100 \%$ & \\
\hline $1994-1998$ & $\begin{array}{l}\text { Programme d'action de relance économique et } \\
\text { sociale (PARESO) }\end{array}$ & 164,934 & $75 \%$ & $100 \%$ \\
\hline $2000-2002$ & Le programme intérimaire post-conflit (PIPC) & 2011,065 & $100 \%$ & $100 \%$ \\
\hline $2005-2007$ & $\begin{array}{l}\text { Document de stratégie de la réduction de la } \\
\text { pauvreté (DSRP-I) }\end{array}$ & 132,72 & $100 \%$ & \\
\hline $2007-2010$ & $\begin{array}{l}\text { Document de stratégie de la réduction de la } \\
\text { pauvreté (DSRP-II) }\end{array}$ & 4513,09 & $100 \%$ & \\
\hline $2009-2013$ & $\begin{array}{l}\text { Plan cadre des nations unies pour l'aide au } \\
\text { développement (PCNUAD) }\end{array}$ & $48.240,788$ & $100 \%$ & \\
\hline
\end{tabular}

II-2- Les structures de gouvernement issues des stratégies de gestion de la diversité sociale des leaders

II-2-1-Les dimensions du leadership « souverain »: Trois dimensions animent la structure du gouvernement de la Cité: l'équivalence organisationnelle (Sagesse), la compensation distributive (Justice), et le contrôle-régulation (Force).

L'équivalence organisationnelle (EO) est le facteur qui caractérise la Sagesse ou la qualité de la décision du leader dans sa stratégie de distribution des rôles (responsabilités) et des tâches (activités) ministériels entre les différentes les tribus. Ce facteur se mesure par la fréquence de la triade tribale dans la composition du gouvernement de la cité. Les gouvernements à forte équivalence organisationnelle sont ceux qui ont un nombre élevé de Ministres égal entre les principales tribus plus au moins un membre d'une tribu minoritaire. Ce sont les structures géocentriques. Les gouvernements à faible équivalence organisationnelle sont ceux qui ont un nombre faible de Ministre équivalent entre les trois principales tribus plus ou moins un membre d'une tribu minoritaire. Ces sont des gouvernements ethnocentriques.

\footnotetext{
${ }^{2}$ D'après Rapports PNUD (2006) : Principaux résultats de l'étude du Ministère à la Présidence chargé du Contrôle d'Etat sur la corruption et la fraude au Congo, p.36, et PNUD (2009). Le Deuxième tableau les valeurs sont en \% du montant du plan.
} 
Tableau $n^{\circ}$ 9: Le gouvernement équiproportionnel de la fédération du Mali (05/04/195920/08/1960)

\begin{tabular}{|l|c|c|}
\hline Ministres & Malien & Sénégalais \\
\hline Président du conseil : Modiko Kéita & $\mathrm{X}$ & \\
\hline Vice-président du conseil : Mamadou Dia & & $\mathrm{X}$ \\
\hline Ministre de la Justice : Boubacar Guèye & & $\mathrm{X}$ \\
\hline Ministre de la Fonction publique : Ousmae Bâ & $\mathrm{X}$ & \\
\hline Ministre des Finances : Doudou Thiam & & $\mathrm{X}$ \\
\hline Ministre des Travaux publics Amadou Mamadou Aw & $\mathrm{X}$ & \\
\hline Ministre de l'Éducation et de la Santé : Abdoulaye Fofana & & $\mathrm{X}$ \\
\hline Total & 4 & 4 \\
\hline
\end{tabular}

La compensation distributive (CD) est le facteur qui caractérise le degré d'équité de la décision dans l'usage harmonieux de l'administration des ressources, notamment de leur répartition entre les différentes parties prenantes. Ce facteur indique la qualité des actes comme le produit des stratégies d'enracinement des dirigeants, surtout des actions d'investissements idiosyncratiques qu'ils entreprennent sur le plan idéologique pour se rendre indispensables aux yeux des membres des tribus, tout en cherchant à s'affranchir, au moins partiellement, de leur contrôle, en vue de conserver une position dominante permettant d'accroître leur liberté d'action et/ou de maximiser des rentes situationnelles. C'est l'écart entre l'effectif du gouvernement et l'effectif absorbé par l'équivalence organisationnelle (EO). Soit :

$$
\mathrm{CD}=\text { effectif du gouvernement }-\mathrm{EO} \text {. }
$$

Plus le nombre de poste non couvert par l'équivalence organisationnelle est élevé, plus la compensation distributive est faible, plus la répartition des ressources est inéquitable, plus la structure gouvernementale est inefficiente. La structure qui en résulte est alternative, ethnocentrique, c'est-àdire fondée essentiellement sur la fragmentation naturelle de la population, base de la préférence tribale.

Le contrôle-régulation $(\mathbf{C R})$ : C'est le facteur qui mesure la qualité de l'équité dans la valorisation des compétences des membres de la diversité. Il traduit la force ou la capacité du leader à assumer la responsabilité de ses actes. Il s'évalue par le rapport entre la compensation distributive et l'équivalence organisationnelle. Soit : $\mathrm{CR}=\mathrm{CD} / \mathrm{EO}$.

II-2-2- Le lien entre le leadership et la configuration structurelle du gouvernement de la Cité: L'étude des stratégies développées par les partenaires dans la gestion de la diversité sociale peut être abordée par l'analyse en composante principale (ACP) des données issues des structures des 41 gouvernements du Congo (annexe $\left.{ }^{\circ} 1\right)$. L'ACP est une méthode statistique des données qui consiste à rechercher les directions de l'espace qui représentent le mieux les corrélations entre $n$ variables aléatoires d'une population. Il s'agit de trouver un axe $u$, issu d'une combinaison linéaire des $X_{n}$, observations, centrées et réduites, telle que la variance du nuage autour de cet axe soit maximale. Ce sous-espace est celui qui explique le maximum d'inertie. Il représente les corrélations entre toutes les variables, et ce en considérant la variance de chaque variable comme égale à 1 , de telle sorte que la variance totale à expliquer soit égale au nombre total de variables. Le principe d'extraction des axes ou des composantes principales consiste initialement à choisir les composantes de façon séquentielle, en fonction de la variance qu'elle explique. Nous pouvons y constater que les deux premiers facteurs représentent $94,50 \%$ de l'information ; alors que la matrice des corrélations des trois dimensions du leadership avec les facteurs principaux (Tableau $\mathrm{n}^{\circ} 10$ ), montre que le troisième facteur ( CR) évolue inversement proportionnel par rapport au facteur EO (avec un coefficient de corrélation $r=-0,608$ ). Durant la période étudiée, la Sagesse évolue en sens inverse de la Force. Le deuxième facteur (CD) est significativement corrélé avec le facteur $1(\mathrm{r}=0,262)$. La Sagesse est faiblement liée à la Justice. Le facteur $2(\mathrm{CD})$ est significativement lié au facteur $3(\mathrm{r}=0,352)$. La Justice est faiblement liée à la Force. Ces liens montrent que ces facteurs sont significatifs pour analyser le modèle de leadership observé. 
Tableau $\mathbf{n}^{\circ}$ 10: La matrice de corrélations

\begin{tabular}{|l|c|c|c|}
\hline Variables & EO & CD & CR \\
\hline EO & $\mathbf{1}$ & 0,262 & $-0,608$ \\
\hline CD & 0,262 & $\mathbf{1}$ & 0,352 \\
\hline CR & $-0,608$ & 0,352 & $\mathbf{1}$ \\
\hline
\end{tabular}

Le tableau $\mathrm{n}^{\circ} 11$, montre que l'axe vertical (F1) qui représente 53,83\% de l'information, oppose deux groupes de structures de gouvernance et de leadership : la structure ethnocentrique qui a un degré de fragmentation tribale élevé et le style géocentrique qui présente un faible degré de fragmentation tribale. Chaque style comprend deux sous formes absolue et relative. Le premier comprend la forme radicale absolue, représentée par le gouvernement de B.KOLELAS (1997) et la forme relative, représentée par le la structure bureaucratique ou marchande du gouvernement de A.MASSAMBADEBAT (1963). Le style géocentrique comprend deux sous formes : la sous forme géocentrique relative, représentée par la structure marchande du gouvernement d'I.MVOUMBA (2005) et la sous forme géocentrique absolue, représentée par la structure technocratique du gouvernement P.LSSOUBA (1963).

Tableau n ${ }^{\circ}$ 11: La matrice des systèmes de gouvernance et de leadership

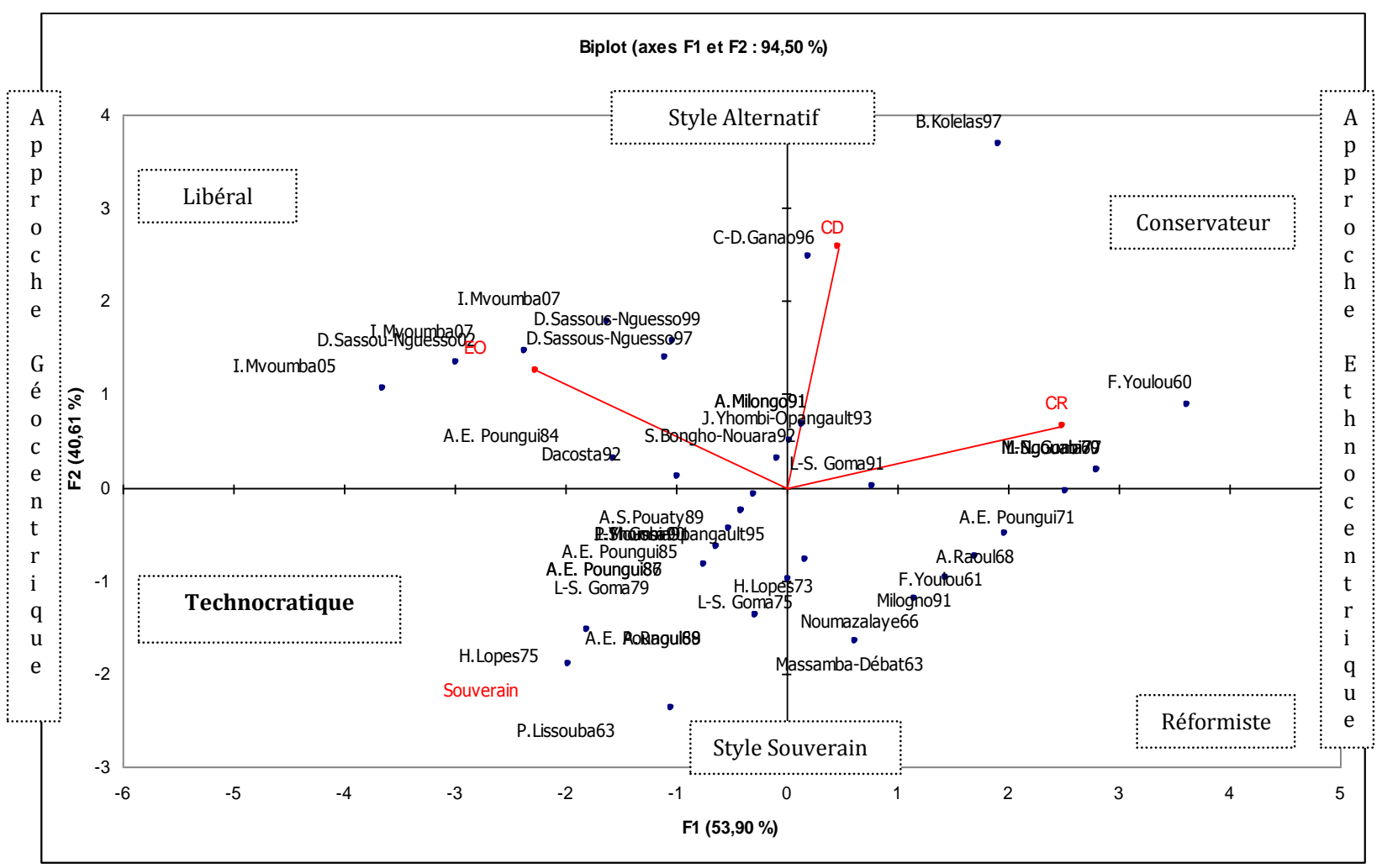

L'axe horizontal (F2) qui représente 40,63\% de l'information, oppose le style souverain (système le plus performant, dans lequel, les trois principaux groupes tribaux ont un nombre égal de ministres plus un), au style alternatif (dans lequel, le nombre de ministres par groupe tribal est inégal. Le premier se décompose en deux sous groupes: le sous groupe de souveraineté relative, représenté par le gouvernement de A.MASSAMBA-DEBAT (1963); et sous groupe de souveraineté absolue, représenté par le gouvernement de P.LISSOUBA (1963). Le deuxième comprend le sous groupe Alternatif relatif, symbolisé par le gouvernement d'I.MVOUMBA (2005) et le sous groupe Alternatif absolu, représenté par le gouvernement de B.KOLELAS (1997). Nous en déduisons quatre configurations : radicale, intégrale, marchande et technocratique.

La structure conservatrice: Elle est fondée sur l'enracinement organisationnel qui consiste à privilégier les domaines ou les personnes que le dirigeant connaît le mieux pour maîtriser les situations ; afin de rendre l'exercice de son pouvoir personnel inévitable. Elle se caractérise par une 
faible équivalence organisationnelle et une forte compensation distributive. Dans le cas étudié, elle s'observe, lorsque la triade tribale est répétée jusqu'à 4 fois plus au moins 25 Ministres supplémentaires provenant majoritairement de la tribu Bakongo. Cette structure est instable, inefficace et inefficiente, car elle répartie très inégalement les rôles et les activités, rassemble moins les tribus et répartit inégalement les ressources entre les parties prenantes. Elle repose sur une gouvernance ethnocentrique absolue. Elle n'est pas souveraine. Ce sont les cas des Gouvernements de F.YOULOU (1960-1963) et B.KOLELAS (1997), caractérisés par le courant le chrétien, libéral et conservateur. La position du gouvernement de M.NGOUABI (1968-1969) sur le graphique, indique le caractère conservateur du courant marxiste léniniste.

La structure libérale: Cette structure est à la fois une bureaucratie traditionnelle (fondée sur les us et les coutumes du leader) et une bureaucratie charismatique (fondée sur le charisme du leader). Elle se manifeste lorsque l'équivalence organisationnelle est très élevée et la compensation distributive est très élevée. Elle est relativement géocentrique, très inefficace, très inefficiente et plus alternative. Dans le cas étudié, cette structure s'observe, lorsque la triade tribale est répétée jusqu'à six fois plus au moins 19 Ministres, venant majoritairement de la tribu majoritaire (Bakongo). Cette structure est budgétivore et inefficace. C'est les cas des gouvernements de D.SASSOU-NGUESSO $(2002,2012)$ et d'I.MVOUBA $(2005,2007,2009)$.

La structure réformiste : C'est celle dans laquelle la gouvernance est fondée sur l'enracinement marchand ou bureaucratique des parties prenantes. L'enracinement marchand consiste pour le dirigeant à fonder la hiérarchie sur les compétences (techniques) des membres, sur leur réputation (réseaux relationnels) et sur leur charisme (titulaire d'un mandat politique sur leurs terres ancestrales), afin de verrouiller les réseaux géopolitiques des relations intersubjectives. Cette structure se caractérise par la recherche des projets coûteux. Elle a un niveau d'équivalence organisationnelle très élevé et un niveau de compensation distributive relativement bas. Elle repose sur une gouvernance géocentrique relative et faiblement alternative intégrant la triade tribale au moins 7 fois plus au moins 16 Ministres Bakongo. Ce sont les cas des gouvernements d'A.E. POUNGUI (1971), A.RAOUL (1968). Ces structures sont très inefficaces et inefficientes. Elles sont représentatives du courant réformiste du socialisme bantou ou socialisme à l'africaine, fondée sur la lutte tribale.

La structure technocratique: Elle est fondée sur l'exacerbation du statut d'actif spécifique des membres, en valorisant leur rôle et leur savoir-faire technique. L'actif spécifique traduit le fait que pour chaque dirigeant, la détention d'un Savoir plus que les autres membres, constitue une condition essentielle de la contractualisation. Cette structure est la plus importante au Congo. Les Gouvernements de P. LISSOUBA (1963-1968) et de H. LOPES (1975) sont les plus représentatifs de ce groupe. Le premier est celui des «technocrates». Le second est celui des «cadres rouges et experts » ou des intellectuels marxistes-léninistes. Ce gouvernement ne durera que les onze mois qui ont suivis la mise en place du plan triennal 1975-1977, avant d'être remplacé par une équipe plus radicale de marxistes-léninistes. D'où la fonction score de la gouvernance $(\Omega)$ obtenue à partir des valeurs des facteurs propres suivantes :

$$
\Omega=1,615 \mathrm{EO}+1,219 \mathrm{CD}+0,166 \mathrm{CR} \text {. }
$$

Cette fonction permet de classer les différentes structures de gouvernement de la Cité dans le graphique suivant (Tableau $\mathrm{n}^{\circ} 12$ ). La structure la plus géocentrique est celle qui a la note la plus basse de 4,53 (LISSOUBA 1963) et la structure la plus ethnocentrique est celle qui a la note la plus élevée de 45,62 (B.KOLELAS 1997). Quatre groupes se distinguent:

- Groupe A, constitué de 2 équipes gouvernementales (4,88\% du total des équipes gouvernementales) qui ont une note allant de 1 à 10 . Ce sont les structures les plus adaptées au modèle traditionnel de gouvernance de la Cité, mais faiblement adaptés au modèle de leadership souverain;

- Groupe B, composé par 20 équipes $(48,78 \%)$ qui ont une note allant de 10 à 20 . Ce sont les structures moyennement adaptées aux modèles traditionnels;

- Groupe C, représenté par 11 équipes $(26,88 \%)$ qui ont une note allant de 20 à 30 . Ce sont les structures faiblement adaptées au modèle traditionnel et fortement adaptées au modèle de leadership traditionnel;

- Groupe D, représenté par 8 équipes $(19,46 \%)$ qui ont une note de plus de 30. Ce sont les structures moins adaptées aux modèles traditionnels de la structure du gouvernement et du leadership.

Ainsi plus de 95,42\% des équipes gouvernementales formées durant la période étudiée, ne sont pas adaptées aux modèles traditionnels de la structure du gouvernement et du leadership. D'où le faible lien entre le style de leadership et la structure du gouvernement se traduit par une instabilité politique et des contre performances économiques chroniques. 
Tableau $\mathrm{n}^{\circ} 12$ : Score des gouvernements

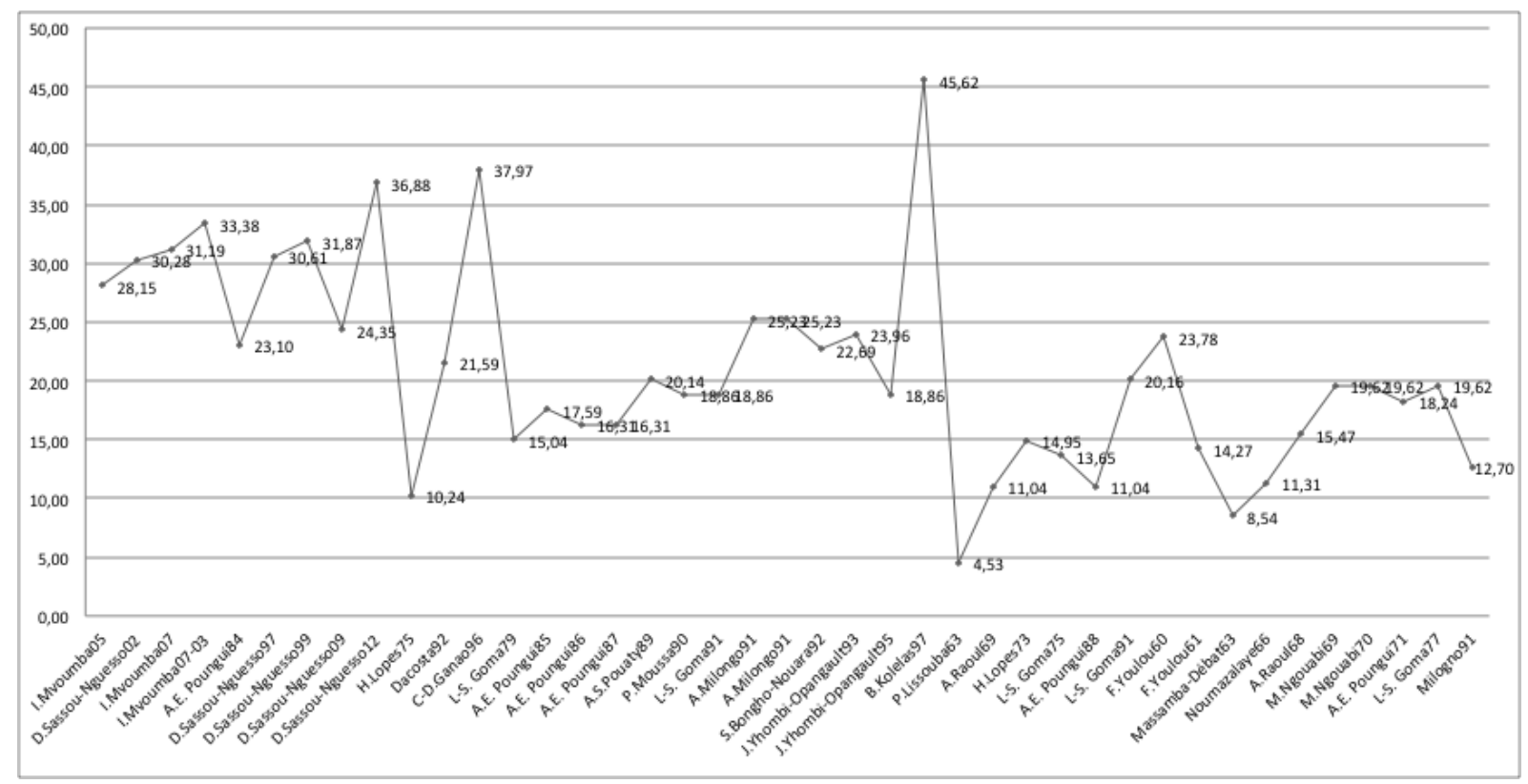

\section{Conclusion}

L'objectif de cet article était de montrer le déterministe du lien entre la structure, le leadership et la performance des organisations à partir du postulat de la primauté de la structure sur les phénomènes. Le cas étudié, montre que l'état programmé de l'avancement de la nation vers une société sans discrimination tribale, se traduit par la réduction progressive des inégalités naturelles entre les tribus majoritaires, en dépit des distorsions institutionnelles, organisationnelles et managériales chroniques du gouvernement de la Cité. Ces distorsions sont liées aux stratégies d'enracinement des membres de la diversité sociale de la population qui évoluent dans un jeu de coopération à somme non nulle où, les rationalités des acteurs sont limitées. Si les leaders des tribus issus de l'ex-royaume du Kongo ont davantage mobilisé une structure équiproportionnelle de type technocratique en phase avec la structure traditionnelle de la Cité, leur leadership est faiblement lié au modèle de leadership mobilisateur traditionnel. Les leaders issus de la chefferie qui ont développé un niveau de leadership mobilisateur élevé, développent faiblement la structure équiproportionnelle du gouvernement de la Cité. Les stratégies mises en œuvre par les dirigeants ont développé davantage la structure d'union nationale que la structure ordinaire, dans un régime plus tôt semi-présidentiel que parlementaire. Leur interaction a conduit à réduire les inégalités dues à la fragmentation tribale naturelle de la population et la naissance d'une oligarchie politique qui concentre le maximum des richesses du pays. La réduction des inégalités individuelles en terme de niveau de vie de la majorité de la population, reste l'enjeu majeur de la gestion de la diversité sociale que les structures transitionnelles doivent consolider sous la pression de la préférence tribale pour atteindre le niveau optimal de la fragmentation sociale, susceptible d'harmoniser le lien entre le leadership, la structure du gouvernement de la Cité, le régime politique et la performance. Cette harmonisation ne peut être conduite que par un dirigeant d'exception dans une structure gouvernementale équiproportionnelle. D'où, le déterministe relatif du lien entre la structure, le leadership et la performance est confirmé par le postulat de la primauté de la structure sur les phénomènes.

\section{Bibliographie}

AOKI M.(2006) : Fondements d'une analyse institutionnelle comparée, Paris, Albin Michel,2006 BATES, R. (1974): «Ethnic competition and modernization in contemporary Africa», Comparative Political Studies, vol. 6, n ${ }^{\circ}$ 4, p. 457

BALANDIER G. (1965) : Le royaume de Kongo du XVIe au XVIIIe siècle. Ed. Hachette, paris, 1965 
BATSIKAMA BA MAMPUYA MA NDAWLA R. (1999): L'Ancien Royaume du Congo et les Bakongo, éd. L'Harmattan, Paris, 1999

BOKILO J.(2012) : La Chine au Congo-Brazzaville : stratégie d'enracinement et conséquences sur le développement en Afrique. Ed. L'Harmattan, Paris, 2012

BOSSUROY T (2006) : «Déterminants de l'identification ethnique en Afrique de l'Ouest », Afrique Contemporaine $\mathrm{n}^{\circ} 220$ p.p. 119 à 126

CHANDLER A. (1989): Stratégies et structures de l'entreprise, Paris, Organisation. Traduction de Strategy and Structure, Cambridge, Mass.: MIT Press, 1962

DEUTSCH, K. (1963): The nerves of government, New York, Free Press, 1963.

DUMEZIL G. (1958): L'idéologie tripartite des Indo-Européens. Latomus, Paris 1958

EASTERLY, W., RITZEN, J. et M. WOOLCOCK (2006): «Social cohesion, institutions, and growth», Economics \& Politics, vol. 18, Issue 2, p. 103-120.

CHARREAUX G. (1997): Le gouvernement des entreprises : Corporate Governance : Théories et faits, Paris, Economica, 1997, p.1

CHARREAUX G., PITOL-BELLIN JP(1997) : "La théorie contractuelle des organisations : une application au conseil d'administration", in Le gouvernement des entreprises: Corporate Governance : Théories et faits, Paris, Economica, 1997, p-p165-192.

GARVEY G.T, SWAN P.L. (1994): «The Economics of corporate governance: Beyond the Marshallian firm », Journal of Corporate Finance, Vol 1, 1994, p.139-174.

GOLEMAN, D., BOYATZIS, R. \& MCKEE A. (2002): L'intelligence émotionnelle au travail

(Primal leadership), Village mondial

D'IRIBARNE P. (1989) : La logique de l'honneur. Ed. Seuil, Paris 1989

HUNTINGTON S.-P.(1968): Political order in changing societies, New Haven, Yale University Press.

LEVI-STRAUSS C. (1958): Anthropologie structurale, Edition Plon, Paris 1958 ; 1973

LOMBARD J. (1967) : Autorités traditionnelles et pouvoirs européens en Afrique Noire. Cahiers de la Fondation Nationale des Sciences Politiques, ${ }^{\circ} 152$ Ed. Armand Colin, 1967, p.p 50-123

RUBINSTEIN M. (2006)) : Le développement de la responsabilité sociale de l'entreprise

Une analyse en termes d'isomorphisme institutionnel, Revue d'Economie Industrielle, ${ }^{\circ} 113, \mathrm{p} .83-105$

MACNEIL I.R. (1974): «Contrats : Adjustements of Longterm Economic Relations under Classical, Neoclassical and Elationnal Contrat Law », Northwestern University Law Review, 72, pp.451-476

MOUAYINI OPOU, E. (2005): Le royaume téké, Études africaines, Édition L'Harmattan, Paris

OKAMBA E.(2010): Gouvernance, une affaire de société: analyse mythiumétrique de la performance, Ed. L'Harmattan, Paris 2010

OKAMBA E. (2012): Ethique du Kébé-kébé et promotion du leadership chez les Mbosi du Congo : le réveil d'Odi, Ed. L'Harmattan, Paris 2012

SHIEIFER A, VISHNY R.W., MORCK R. (1989): «Alternative mechanisms for corporate control », American Economic Review,79,N4,1989.

WEBER M. (1904) : L'Éthique protestante et l'esprit du capitalisme, Gallimard, 2004 


\section{Annexe $n^{\circ}$ 1: L'évolution de la structure du Gouvernement du Congo de 1960 à nos}

jours

\begin{tabular}{|c|c|c|c|c|c|c|c|c|c|c|c|}
\hline & Début & Fin & $\begin{array}{c}\text { Durée du } \\
\text { mandat (ans) }\end{array}$ & Tribu & Effectif & $\begin{array}{l}\text { (E)Fréquence } \\
\text { de la Triade } \\
\text { tribale (EO) }\end{array}$ & $\begin{array}{c}\text { Taille après } \\
\text { triade }(\mathrm{CD}) \\
=\mathrm{E}-\mathrm{EO}\end{array}$ & $\begin{array}{r}\text { Indice de } \\
\text { Contrôle } \\
(\mathrm{CR})=\mathrm{EO} / \\
\mathrm{CD}\end{array}$ & Parti & $\begin{array}{c}\text { Ministre } \\
\text { d'Etat }\end{array}$ & Profession \\
\hline F.YOULOU60 & $15 / 08 / 60$ & $15 / 08 / 63$ & 3,00 & Bakongo & 19,00 & 1,00 & 16,00 & 16,00 & $\begin{array}{l}\text { UDD } \\
\text { IA } \\
\end{array}$ & 2 & Prêtre \\
\hline F.YOULOU61 & $02 / 03 / 61$ & $15 / 08 / 63$ & 2,45 & Bakongo & 12,00 & 1,00 & 9,00 & 9,00 & $\begin{array}{l}U D D \\
I A\end{array}$ & 2 & Prêtre \\
\hline A.MASSAMBA-DEBAT63 & $19 / 08 / 63$ & $04 / 09 / 63$ & 0,04 & Bakongo & 8,00 & 1,00 & 5,00 & 5,00 & $M N R$ & 1 & Enseignant \\
\hline P.LISSOUBA63 & $24 / 12 / 63$ & $15 / 04 / 66$ & 2,31 & Téké & 7,00 & 2,00 & 1,00 & 0,50 & $M N R$ & 3 & Enseignant \\
\hline A.NOUMAZALAY66 & $06 / 05 / 66$ & $12 / 01 / 68$ & 1,69 & Sangha & 10,00 & 1,00 & 7,00 & 7,00 & $M N R$ & 0 & Administrateur \\
\hline A.RAOUL68 & $20 / 08 / 68$ & $21 / 06 / 69$ & 0,84 & Bakongo & 13,00 & 1,00 & 10,00 & 10,00 & $M N R$ & 1 & Militaire \\
\hline A.RAOUL69 & $21 / 06 / 69$ & $31 / 12 / 69$ & 0,53 & Bakongo & 12,00 & 2,00 & 6,00 & 3,00 & $P C T$ & 1 & Militaire \\
\hline M.NGOUABI69 & $31 / 12 / 69$ & $04 / 01 / 70$ & 0,01 & Mbosi & 16,00 & 1,00 & 13,00 & 13,00 & $P C T$ & 1 & Militaire \\
\hline M.NGOUABI7O & $04 / 01 / 70$ & $16 / 12 / 71$ & 1,95 & Mbosi & 16,00 & 1,00 & 13,00 & 13,00 & $P C T$ & 2 & Militaire \\
\hline A.E. POUNGUI7I & $16 / 12 / 71$ & $28 / 07 / 73$ & 1,62 & Bakongo & 15,00 & 1,00 & 12,00 & 12,00 & $P C T$ & 1 & Administrateur \\
\hline H.LOPES73 & $28 / 07 / 73$ & $08 / 01 / 75$ & 1,45 & Téké & 15,00 & 2,00 & 9,00 & 4,50 & $P C T$ & 0 & Administrateur \\
\hline H.LOPES75 & $08 / 01 / 75$ & $19 / 12 / 75$ & 0,95 & Téké & 15,00 & 4,00 & 3,00 & 0,75 & $P C T$ & 0 & Administrateur \\
\hline L-S. GOMA75 & $19 / 12 / 75$ & $05 / 04 / 77$ & 1,30 & Bakongo & 14,00 & 2,00 & 8,00 & 4,00 & $P C T$ & 0 & Militaire \\
\hline L-S. GOMA75 & $05 / 04 / 77$ & $04 / 05 / 79$ & 2,08 & Bakongo & 16,00 & 1,00 & 13,00 & 13,00 & $P C T$ & 0 & Militaire \\
\hline L-S. GOMA79 & $04 / 05 / 79$ & $07 / 05 / 84$ & 5,01 & Bakongo & 17,00 & 3,00 & 8,00 & 2,67 & $P C T$ & 0 & Militaire \\
\hline A.E. POUNGUI84 & $07 / 08 / 84$ & $07 / 12 / 85$ & 1,33 & Bakongo & 27,00 & 5,00 & 12,00 & 2,40 & $P C T$ & 0 & Administrateur \\
\hline A.E. POUNGUI85 & $07 / 12 / 85$ & $10 / 12 / 86$ & 1,01 & Bakongo & 19,00 & 3,00 & 10,00 & 3,33 & $P C T$ & 0 & Administrateur \\
\hline A.E. POUNGUI86 & $10 / 12 / 86$ & $20 / 08 / 87$ & 0,69 & Bakongo & 18,00 & 3,00 & 9,00 & 3,00 & $P C T$ & 0 & Administrateur \\
\hline A.E. POUNGUI87 & $20 / 08 / 87$ & $30 / 07 / 88$ & 0,95 & Bakongo & 18,00 & 3,00 & 9,00 & 3,00 & $P C T$ & 0 & Administrateur \\
\hline A.E. POUNGUI88 & $30 / 07 / 88$ & $12 / 08 / 89$ & 1,04 & Bakongo & 12,00 & 2,00 & 6,00 & 3,00 & $P C T$ & 0 & Administrateur \\
\hline A.S.POUATY89 & $12 / 08 / 89$ & $03 / 12 / 90$ & 1,31 & Bakongo & 21,00 & 3,00 & 12,00 & 4,00 & $P C T$ & 0 & Administrateur \\
\hline P.MOUSSA90 & $03 / 12 / 90$ & $08 / 01 / 91$ & 0,10 & Mbosi & 20,00 & 3,00 & 11,00 & 3,67 & $P C T$ & 0 & Administrateur \\
\hline L-S. GOMA91 & $08 / 01 / 91$ & $08 / 06 / 91$ & 0,41 & Bakongo & 20,00 & 3,00 & 11,00 & 3,67 & $P C T$ & 0 & Militaire \\
\hline L-S. GOMA90 & $08 / 01 / 90$ & $14 / 01 / 91$ & 1,02 & Bakongo & 19,00 & 2,00 & 13,00 & 6,50 & $P C T$ & 0 & Militaire \\
\hline A.MILONGO91 & $08 / 06 / 91$ & $30 / 12 / 91$ & 0,56 & Bakongo & 25,00 & 3,00 & 16,00 & 5,33 & $\begin{array}{l}\text { Néan } \\
t \\
\end{array}$ & 0 & Administrateur \\
\hline A.MILONGO91 & $30 / 12 / 91$ & $26 / 01 / 92$ & 0,07 & Bakongo & 25,00 & 3,00 & 16,00 & 5,33 & $\begin{array}{l}\text { Néan } \\
t \\
\end{array}$ & 0 & Administrateur \\
\hline A.MILONGO92 & $26 / 01 / 92$ & $07 / 09 / 92$ & 0,62 & Bakongo & 11,00 & 1,00 & 8,00 & 8,00 & $\begin{array}{l}\text { Néan } \\
t \\
\end{array}$ & 0 & Administrateur \\
\hline S.BONGHO NOUARA92 & $02 / 09 / 92$ & $25 / 12 / 92$ & 0,31 & Mbosi & 23,00 & 3,00 & 14,00 & 4,67 & $\begin{array}{l}\text { UPA } \\
\text { DS }\end{array}$ & 3 & Administrateur \\
\hline DACOSTA92 & $25 / 12 / 92$ & $23 / 06 / 93$ & 0,49 & Bakongo & 24,00 & 4,00 & 12,00 & 3,00 & $\begin{array}{l}\text { UPA } \\
\text { DS }\end{array}$ & 3 & Militaire \\
\hline $\begin{array}{l}\text { J;YHOMBI- } \\
\text { OPANGAULT93 }\end{array}$ & $24 / 06 / 93$ & $22 / 01 / 95$ & 1,58 & Mbosi & 24,00 & 3,00 & 15,00 & 5,00 & URD & 3 & Militaire \\
\hline $\begin{array}{l}\text { J;YHOMBI- } \\
\text { OPANGAULT95 }\end{array}$ & $22 / 01 / 95$ & $27 / 08 / 96$ & 1,60 & Mbosi & 20,00 & 3,00 & 11,00 & 3,67 & URD & 3 & Militaire \\
\hline C-D.GANAO96 & $27 / 08 / 96$ & $08 / 07 / 97$ & 0,86 & Téké & 37,00 & 4,00 & 25,00 & 6,25 & UFD & 3 & Enseignant \\
\hline B. KOLELAS97 & $08 / 09 / 97$ & $15 / 10 / 97$ & 0,10 & Bakongo & 41,00 & 3,00 & 32,00 & 10,67 & $\begin{array}{l}M D C \\
C I \\
\end{array}$ & 4 & Administrateur \\
\hline D.SASSOU-NGUESSO 97 & $02 / 11 / 97$ & $12 / 01 / 99$ & 1,19 & Mbosi & 33,00 & 5,00 & 18,00 & 3,60 & $P C T$ & 0 & Militaire \\
\hline D.SASSOU-NGUESSO 99 & $12 / 01 / 99$ & $18 / 08 / 02$ & 3,60 & Mbosi & 34,00 & 5,00 & 19,00 & 3,80 & $P C T$ & 0 & Militaire \\
\hline D.SASSOU-NGUESSOO2 & $18 / 08 / 02$ & $07 / 01 / 05$ & 2,39 & Mbosi & 38,00 & 8,00 & 14,00 & 1,75 & $P C T$ & 0 & Militaire \\
\hline I.MVOUMBA05 & $07 / 01 / 05$ & 03/03/07 & 2,15 & Bakongo & 38,00 & 9,00 & 11,00 & 1,22 & PCT & 0 & Administrateur \\
\hline I.MVOUMBA07 & $03 / 03 / 07$ & $30 / 12 / 07$ & 0,83 & Bakongo & 37,00 & 6,00 & 19,00 & 3,17 & PCT & 0 & Administrateur \\
\hline I.MVOUMBA & $30 / 03 / 07$ & $15 / 09 / 09$ & 2,47 & Bakongo & 37,00 & 7,00 & 16,00 & 2,29 & $P C T$ & 4 & Administrateur \\
\hline D.SASSOU-NGUESSO & 15/09/09 & $25 / 09 / 12$ & 3,03 & Mbosi & 38,00 & 5,00 & 23,00 & 4,60 & $P C T$ & 4 & Militaire \\
\hline D.SASSOU-NGUESSO & $15 / 09 / 12$ & & & Mbosi & 38,00 & 2,00 & 32,00 & 16,00 & $P C T$ & 5 & Militaire \\
\hline
\end{tabular}

$\mathrm{EO}=$ Equivalence Organisationnelle, $\mathrm{CD}=$ Compensation Distributive, $\mathrm{CR}=$ Contrôle Régulation

En Italique : Gouvernement de type Union Nationale : en non italique : Gouvernement Ordinaire

UDDIA = l'Union pour la Défense Démocratique des Intérêts Africains; $P C T=$ Parti Congolais du Travail, UPADS = l'Union Panafricaine pour la Démocratie Sociale

Sources : D'après : http://www.brazza.info/pages/histoire.htm; http://perspective.usherbrooke.ca/bilan/servlet/BMGvt?codePays=COG. 